Erratum

\title{
Therapeutic target values in oral anticoagulation - Justification of Dutch policy and a warning against the so-called moderate-intensity regimens
}

\section{E. A. Loeliger}

Afdeling Hematologie, Academisch Ziekenhuis, 2300 RC Leiden, The Netherlands

Ann Hematol (1992) 64: 60-65

Owing to an unfortunate error, some small squares were printed in this article. In their place, the symbol $>$ should have appeared. 